\title{
Software, objects, and home space
}

\section{Martin Dodge}

Department of Geography, School of Environment and Development, University of Manchester, Oxford Road, Manchester M13 9PL, England; e-mail: m.dodge@manchester.ac.uk

\section{Rob Kitchin}

Department of Geography, National University of Ireland, Maynooth, Co. Kildare, Ireland; e-mail: rob.kitchin@nuim.ie

Received 31 January 2008; in revised form 8 August 2008; published online 6 April 2009

\begin{abstract}
Through a series of interrelated developments, software is imbuing everyday objects with capacities that allow them to do additional and new types of work. On the one hand, objects are remade and recast through interconnecting circuits of software that make them machine readable. On the other, objects are gaining calculative capacities and awareness of their environment that allow them to conduct their own work, with only intermittent human oversight, as part of diverse actant networks. In the first part of the paper we examine the relationship between objects and software in detail, constructing a taxonomy of new types of coded objects. In the second part we explore how the technicity of different kinds of coded objects is mobilised to transduce space by considering the various ways in which coded objects are reshaping home life in different domestic spaces.
\end{abstract}

\section{Introduction}

A number of analysts have recently argued that software is increasingly making a difference to the constitution and production of everyday life, in large part because it alters the conditions through which space is beckoned into being (see Beer, 2007; Crang and Graham, 2007; Dodge and Kitchin, 2005a; Galloway, 2004; Graham, 2005; Mitchell, 2005; Thrift and French, 2002). In previous work we produced a provisional taxonomy of the various ways in which software is becoming embedded in the world (Dodge and Kitchin, 2005a). We detailed that coded objects are items "that use code to function or permanently store digital data that cannot be accessed without software" (page 163); coded infrastructures are distributed "networks that link coded objects together and infrastructure that is monitored and regulated, either fully or in part, by code" (page 163); coded processes "refer to the transaction and flow of digital data across coded infrastructure" (page 164); and coded assemblages consist of the interlinking of coded objects, infrastructures, and processes to produce dense networks that support particular environments or enterprises, such as an airport (cf Dodge and Kitchin, 2004). In our analysis we traced, in broad terms, how these coded objects, infrastructures, processes, and assemblages work together to shape sociospatial life with respect to different domains of everyday living (work, travel, consumption, and the home). However, we provided little in-depth analysis of the nature of coded objects, infrastructures, processes, and assemblages themselves.

In this paper we more fully explore the relationship between software, objects, and material spatiality, examining how the embedding of microprocessors and software algorithms into the objects people use to undertake daily domestic tasks is transforming these objects, imbuing them with capacities that allow them to do additional and new types of work in the world as part of diverse actant networks. Objects, as we will illustrate, are gaining additional competencies: to sense their environment, to record their own use, to take over aspects of decision making from their human owners; 
and, in some cases, are enrolled as nodes in the emerging 'Internet of things'(1) (Schoenberger, 2002). In particular, we are interested in how coded objects beckon particular kinds of space into being through their work in the world.

The space we focus on in this paper is the home, and how new coded objects are shaping domestic practices in Western societies: on the one hand, augmenting the work of existing everyday electrical and electronic technologies in producing particular spatialities and, on the other, establishing new sociospatial arrangements. To that end, the paper is divided into two broad sections. In the first part we discuss the nature of coded objects - that is, objects for which software is a component essential to their operation-detailing the difference that software makes and providing a functional taxonomy of such objects. In the second part of the paper we discuss how various coded objects beckon homes into being, thinking through how the agency of software is beginning to reshape domestic life. While much of this reshaping remains banal and occurs in subtle and often hard to discern ways, it is socially significant, we argue, because it marks a juncture point in the production of the so-called 'technological unconscious' - "the surface on which life floats" (Thrift, 2004, page 584). In so doing, we seek to contribute to the literatures on software and space, material geographies, and geographies of the home, and to bring them into conversation with each other. Importantly, we focus on the messily arranged here and now, rather than imagine the supposed 'smart home' of the future.

\section{Code and (domestic) objects}

Material objects are becoming coded in two ways: firstly, through 'external' processes of identification and linkage, and, secondly, through the 'internal' embedding of software. To consider the first process: since the late 1970s and the widespread application of barcodes to mass-produced consumer goods, objects have increasingly become machine readable through the rapid and reliable reading of identification numbers placed on them. Such identification technologies include a range of different printed barcodes and the growing use of radio frequency identity (RFID) tags (cf Dodge and Kitchin, 2005b) which, when combined with appropriate information infrastructures (for identification-number allocation and specifying product classification formats), can be consistently matched to information held in an organisation's database to reveal the identity of the object and other associated properties (such as batch number, manufacture date, and shipping history). ${ }^{(2)}$ As such, it is now increasingly possible to track objects systematically through space and time in ways that were previously impossible, especially given the rise of unique indexing systems, for example, RFID being promulgated by the EPCglobal corporation, and the widespread deployment of sensors and scanners cable of reading such indexically labelled objects, making tagged goods individually recognisable anywhere.

The near-universal application of these kinds of identification and tracking technologies to manufactured goods has had a major impact on the workings of Western consumer societies, not least in enhancing the operational capacity and logistical

(1) Enrolment in the 'Internet of things', using technologies of RFID tags and electronic product code (EPC) databases, makes objects uniquely identifiable, inherently trackable, and potentially communicative of their status across distributed networks. In much the same way that the location of a website can be 'looked-up' through its unique domain name from anywhere on the Internet, it is envisaged that the 'Internet of things' will facilitate the same for any tagged object. It is essentially a universal indexing for anything and everything that matters and a mechanism by which objects can connect to, transfer, and process information with each other and people (cf Dodge and Kitchin, 2005b).

${ }^{(2)}$ For example, the ubiquitous twelve digit universal product code (UPC) system found on retail products. 
efficiency of retail supply chains. Indeed, such complex distributed and distanciated chains are only possible with effective identification technologies for tracking the movement of goods coordinated within an ERP system. ${ }^{(3)}$ Yet, typically, this has remained behind the scenes and often at several steps removed from the home itself. The banal power of the UPC barcode, for example, may have reconfigured how we buy products, but it has had virtually no effect on what we do with these products inside the home. However, emerging RFID-based technologies, according to some predictions, will have a much greater effect on domestic routines inside the home. 'Smart' packaging using RFID tags will make retail products automatically readable by domestic appliances: for example, a ready meal will 'tell' a microwave how long it should be cooked for; the fridge will be able identify when food goes out of date and warn the household; and a new cashmere cardigan will instruct a washing machine how it should be washed so as not to shrink. The goal, as with much previous domestic technology, is to increase convenience by delegating more components of routine tasks to the machine, in this case through the 'invisible' exchange of unique identification data which enables the software in an appliance to work appropriately without explicit instruction from a human.

To what degree these archetypal 'smart kitchen' scenarios based on automated reading of RFID tags become a reality remains somewhat in the future, with many technologists suggesting that they will be standard aspects of a pervasive computing society (see below). Yet, bound up with the promises of greater convenience and more orderly domestic routines is the capacity to make formerly hidden and unrecorded actions newly visible to other, external organisations and to eliminate anonymity from the consumption of mass products, because every time an RFID tag is queried it leaves behind a log. What this means, in the context of a household, is a change in (1) the ontological status of each product that is indexed, with it being knowable in new ways in terms of what information is attributable to it, and can be generated with respect to it - ranging from purchasing information through to a detailed usage trail and, eventually, disposal (so it is not just $a$ bottle of whisky in a household's rubbish bin but the bottle of whisky purchased for $£ 15.99$ on the $15 / 12 / 07$, at $19.54 \mathrm{pm}$ in the Whistlestop shop, King's Cross Station by A N Other); (2) the epistemological status of each product, with it being useable in new ways, and able to do additional work in the world, and to be worked upon by other entities such as information systems. As such, RFID 'smart' tagging opens up the spectre of a new frontier of potentially invasive surveillance straight into the private sanctuary of the home (cf Albrecht and McIntyre, 2005).

Second, in contrast to machine-readable objects, which simply participate externally in the 'Internet of things', there are objects that have code physically embedded into their material form, altering 'internally' their relations with the world. In such objects software is used on the one hand to enhance or augment the functional capacity of what were previously 'dumb' objects, enabling them to sense something of their environments and to perform different tasks, or the same tasks more efficiently; or to be 'plugged into' new distributed networks that afford some value-added dimension, such as data exchange on how they are used. On the other hand, code is used to underpin the design and deployment of new classes of objects, particularly mobile devices (such as PDAs - personal digital assistants, MP3 players, Satnav) which,

(3) Enterprise resource planning (ERP) systems are vital database systems that tie together many functions and activities of large businesses and institutions, enabling coordination of processes and generating information for day-to-day management and long-term planning. The market leader in this field is SAP, whose software is largely unseen but is a pivotal component of globalised capitalism (cf Pollock and Williams, 2008). 


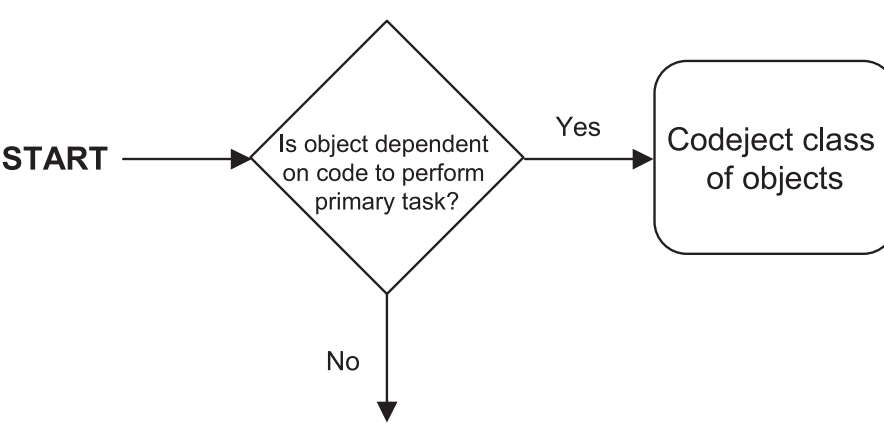

Peripherally

coded object logject (NL)

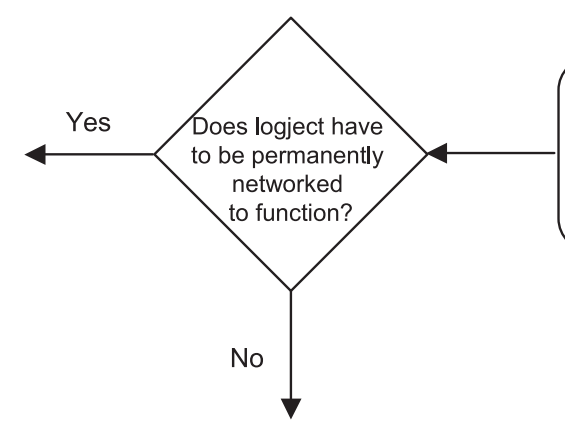

Permeable logject (PL)

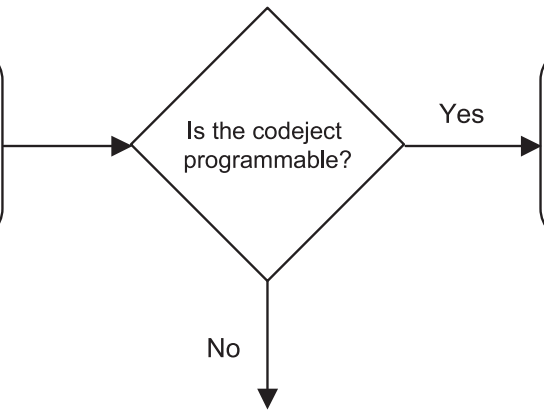

Unitary codeject class of objects

Hard codeject (HC)

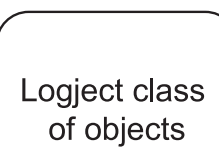

No

oes codeject record

its activity and reuse

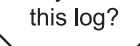

this $\log$ ?

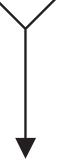

Sensory codeject (SC)

Figure 1. A decision tree detailing the key distinguishing characteristics used in our taxonomy of coded objects. 
in some cases, replace analogue equivalents (diaries and Filofaxes, personal tape and compact disc-CD_stereos, paper maps and street atlases) or undertake entirely new tasks. The embedding of software significantly increases the technicity of an object [its capacity to do meaningful work in the world (Mackenzie, 2003)].

In thinking through the relationship between code and its embedding into objects, we have used the decision-making process detailed in figure 1 to subdivide objects into two general types based on the level of significance of software to the primary function(s) of an object. (4) 'Peripherally coded objects' are objects in which software has been embedded, but such code is not essential to their use (that is, if the software fails they still work as intended, but not as efficiently, cost effectively, or productively). 'Codejects', on the other hand, are dependent upon code to function-the object and its code are thoroughly interdependent and nonseparable (hence our conjoining of the terms 'code' and 'object' to denote this mutual interdependence). Codejects can be further subdivided into three main classes on the basis of their programmability, interactivity, remembering capacity, their ability for anticipatory action in the future based on previous use, and relational capacities.

1. Hard codejects rely on code to function, but are not programmable and therefore have low levels of interactivity.

2. Unitary codejects are programmable, exhibit some level of interactivity (although this is limited and highly scripted), and do not record their work in the world. They can be divided into two classes - closed codejects and sensory codejects-depending on whether they sense and react to the world around them.

3. Logjects are objects which have an 'awareness' of themselves and of their relations with the world and which, by default, automatically record aspects of those relations in logs that are stored and reused in the future. Logjects often have high levels of interactivity and multifunctionality. Logjects can be divided into two classes based on their capacity to work independently of wider networks: permeable logjects and networked logjects.

\section{Peripherally coded objects}

Coded objects are objects in which code has been embedded but in which this software is incidental to the primary function of the object. There are relatively few such objects and, in most cases, the code merely augments their use but is by no means essential to their functioning. Often, the presence of code is merely adornment that serves the purpose of product marketing: to differentiate it from predecessors, or as a token of added value. For example, a gas cooker might have a digital clock embedded in it, but if this timer ceases to function the cooker will continue to cook food. Similarly, an exercise bike might have a device that digitally displays the speed at which the cyclist is pedalling, but if this ceases to work the bike still enables exercise to take place. In both cases the code does little more than augment the use of the object-by enabling the chef to know how long a dish has been cooking, and the cyclist to know his or her speed. Both are simply digital replacements for analogue technology.

\section{Hard codejects}

Hard codejects have firmware ${ }^{(5)}$ embedded into them which is essential for their functioning. Firmware consists of a defined set of routines being stored permanently in read-only memory on a device, rather than being enacted through an executable

(4) Clearly, asking different questions would have produced a different taxonomy, but we feel that the distinctions we draw are useful for thinking through how the embedding of software into objects gives them different capacities.

(5) Firmware is software embedded onto the microprocessor to provide low-level control functions for the hardware. 
programme that can be accessed and interfaced with. Examples include a USB memory stick and basic SCADA (supervisory control and data acquisition) systems in which code monitors and controls an industrial process. Both rely on code, but the functionality of the code is hardwired and predetermined.

\section{Unitary codejects}

Unlike hard codejects, unitary codejects are programmable to some degree and therefore exhibit some degree of interactivity: users are able to control some aspects of the functionality of the object and to instruct it what to do and when. They, along with logjects, exhibit (1) liveness - a feeling that there are infinite possibilities to explore; (2) plasticity - the person interacting with the codeject feels that he or she can push without breaking the system; (3) accretion - the computation improves and evolves with use; and (4) interruption - computation is open to unpredictable input and can react to it (Murtaugh, 2008).

In broad terms, unitary codejects ${ }^{(6)}$ can be divided into those which function independently of their surroundings (closed codejects) and those which are equipped with some kinds of sensors which enable the object to react meaningfully to particular variables in the immediate environment (sensory codejects).

Closed codejects can include digital clocks, and some audiovisual equipment such as radios, and CD and DVD players. Code is vital to the functioning and performance of each of these items, but the object executes its task independently of the world around it. Each is programmable to some degree - the time can be adjusted, stopwatch operated, alarm set, programme record times fixed, the order of tracks selected-but generally they have circumscribed functions and limited latitude to operate automatically.

Sensory codejects have some awareness of their environment and react automatically to some kind of external stimulus: common domestic examples include a heating/air conditioner control unit; a washing machine that is monitored and controlled by software; and a digital camera and storage card. The heating/air

Table 1. Hotpoint washer-dryer error codes that are displayed by software to the user. These codes give a partial indication of the range of conditions that the appliance software monitors (source: Hotpoint Service website-Help Centre, 9 November 2007, http://www.hotpointservice. co.uk/hs/pages/content.do?keys=FAQ:ERROR_CODES).

Fault codes for LCD EVO1 Washing Machines and Washer Dryers

F01 - Short circuit motor triac-Book a service engineer.

F02-Motor jammed tacho detached-Book a service engineer.

F03 - Wash thermistor open/short circuit-Book a service engineer.

F04-Pressure switch jammed on empty-Book a service engineer.

F05-Pressure switch jammed on full-Book a service engineer.

F06-Program selector error-Book a service engineer.

F07-Heater relay stuck - Book a service engineer.

F08-Heater relay cannot be activated-Book a service engineer.

F09-Incompatible eeprom-Book a service engineer.

F10-Pressure switch not sensing correctly-Book a service engineer.

F11-Pump cannot be activated-Book a service engineer.

F12-Communication error-Book a service engineer.

F13-Dryer fan or dryer thermistor faulty-Book a service engineer.

F14-Dryer element faulty-Book a service engineer.

F15-Dryer element relay faulty-Book a service engineer.

H20 - Not filling. Check tap, hose and inlet valves.

LOCKED-Check interlock-Book a service engineer.

(6) We term them 'unitary' because it is self-contained, having everything it needs within its material form and does not record beyond the immediate cycle of use. 
conditioning unit is equipped with a digital thermostat and timer that is 'aware' of the time/date and senses the surrounding temperature. Simple software algorithms react according to temperature measurements in relation to people's preset requirements. Similarly, software embedded in the washing machine will monitor multiple contextual parameters, such as door lock, load weight, and water temperature necessary for safe and effective operation without human oversight (see table 1). The digital camera captures an image of the world by means of a CCD sensor ${ }^{(7)}$ and measures light levels, adjusts the aperture setting accordingly, and the lens movement for autofocusing, as well as monitoring remaining battery life and available storage space.

\section{Logjects}

Logjects differ from unitary objects in that they also record their status and usage and, importantly, can retain these logs even when deactivated and utilise them when reactivated. In key ways these logs can have a bearing on the ongoing operation of the object and its relations with people or wider processes. Furthermore, part of their functionality is externalised, lying beyond the immediate material form of the object.

We derive the term 'logjects' from Bleecker's (2006) notion of a blogject (where, for us, a blogject is one type of logject). Bleecker defines a blogject as an emerging class of objects that generates a kind of blog of its own use and has the capability automatically to initiate exchanges of socially meaningful information- "it is an artefact that can disseminate a record of experience to the web" (Nova and Bleecker, 2006, no pagination). Bleecker (2006, page 6, original emphasis) characterises blogjects as objects that: (a) can "track and trace where they are and where they've been"; (b) "have self-contained (embedded) histories of their encounters and experiences" (rather than indexical histories); and (c) "have some form of agency - they can foment action and participate; they have an assertive voice within the social web." Blogjects are things that can 'do' meaningful social acts where their actions shape how people think about and act in the world; they "participat[e] within the Internet of social networks" (Bleecker, 2006, page 2). Here, Bleecker is very much interested in only certain kinds of software-enabled objects which produce streams of information very much like humans' writing blogs, thus contributing to the "ecology of networked publics - streams, feeds, trackbacks, permalinks, wiki inscriptions and blog posts" (2006, page 9). He is very careful to delineate blogjects as political actants which contribute to debates by providing socially meaningful information, rather than being coded objects which log their use and communicate and/or analyse that data across distributed networks.

While Bleecker's notion of a blogject has some conceptual utility, for us, it is one form of logging object in a much larger sociotechnical ecology of logjects. We broadly define a 'logject' as an object that monitors and records its own use in some fashion. More specifically, and expanding on Bleecker, it is: (1) uniquely indexical; (2) has awareness of its environment and is able to respond to changes in that environment that are meaningful within its functional context; (3) traces and tracks its own usage in time and/or space; (4) records that history; (5) can communicate that history across a network for analysis and use by other agents (objects and people); (6) can use the data it produces to undertake what we have previously termed 'automated management' (Dodge and Kitchin, 2007a) - automated, automatic, and autonomous decisions and actions in the world without human oversight - and to effect change through the "consequences of their assertions" (Bleecker, 2006, page 9); and (7) is programmable

(7) A charge-coupled device (CCD) is a sensor that converts light into continuous electrical charge. At the edge of the CCD sensor an analogue-to-digital converter then transforms the electrical charge into a digital form. 
and thus mutable to some degree (that is, it is possible to adjust settings, update parameters, and to download new firmware). Logjects then enable the kinds of unobtrusive machine-to-machine, machine-to-person and person-to-machine exchanges that are a fundamental trait of pervasive computing and are diverse in their nature.

\section{Permeable logjects}

Permeable logjects consist of relatively self-contained units, such as an MP3 player, a PDA, or a satnav, all of which have the potential to be connected to wider networks. Such devices trace and track their usage by default, recording this data as an embedded history; are programmable in terms of configurable settings and list creation (eg play lists of songs, diary entries, and route itineraries); perform operations in automated, automatic, and autonomous ways; and engender socially meaningful acts such as entertaining, remembering an important meeting, and helping an individual to travel between locations. These devices work to relieve the cognitive burden of routine tasks on people who use them, and help to reduce the risks and consequences of unexpected events. Unlike a networked logject, all essential capacities are held locally and primary functionality does not require network connection to operate. That said, data (eg music, diary entries, or map files) and software must be downloaded onto the machine at some point; and GPS (global positioning system) works by receiving (though not exchanging) radio signals from satellites (hence they are permeable). Moreover, these devices are connectable to wider networks so that information can be uploaded and exchanged with other devices (via Bluetooth wireless transmission, for example) and updates in firmware downloaded-although typically this is not automatic, and sometimes requires considerable human intervention (what might be classed 'digital housework' - for example, syncing a PDA or MP3 player). The uploaded information can be processed and analysed in relation to other usage, thus providing added value. The aggregate social significance of such objects is impossible to estimate, but they are used to solve all manner of domestic problems billions of times a day.

\section{Networked logjects}

Networked logjects do not function without continuous access to other technologies and networks. In particular, because they need constant two-way data exchange, they are reliant on access to a distributed communication network to perform their primary function. Such logjects track, trace, and record their usage locally but, because of memory issues, the necessity of service monitoring/billing, and in some cases a user's ability to erase or reprogram such objects, their full histories are also recorded externally to its immediate material form. Some networked logjects are relatively fixed in the environment (for example, satellite/cable television control boxes, home-security monitoring systems) and others are inherently mobile (mobile telephones, telematically monitored vehicles) and use a range of communication technologies - such as GSM (global system for mobile communications), Wifi (wireless fidelity), Bluetooth - to maintain a network connection. Mobile networked logjects continuously search for connectivity and can respond automatically and autonomously to the network conditions. For example, a mobile phone reacts automatically to incoming calls by sounding the ringtone; switches to the answer service if the call is unanswered; and alerts the owner that a call was missed and/or a message is waiting for them. 


\section{Coded objects and the making of homes}

"As a space of belonging and alienation, intimacy and violence, desire and fear, the home is invested with meanings, emotions, experiences and relationships that lie at the heart of human life."

Blunt and Varley (2004, page 3)

"When it is not only 'us' but also our 'things' that can upload, download, disseminate and stream meaningful-making stuff, how does the way in which we occupy the physical world become different?"

Bleecker (2006, page 10)

Work across the social sciences documents how the home is a complex set of social and material relations (see Blunt and Dowling, 2006; Hitchings, 2004; Mallett, 2004; Miller, 2001), and a site of continual technological adoption. Home is a dwelling space in which important lived experiences take place, providing a locus for the fundamental aspects of daily social reproduction (eating, personal care, relaxation and sleep, and so forth). Home is also central to human psychological well-being; a place of familial relations and emotional ties; a place for personal life and privacy from others; a place with layers of memories and meanings of past; a sanctuary which offers security and safety from the wider world. ${ }^{(8)}$ Home is important then, not least because we spend most of our time 'at home'. (9)

In contrast to static and teleological notions of a home, we would view the home as the product of a diverse range of relational and contingent processes. A great deal of emotional, physical, and monetary effort is expended in the maintenance of the physical dwelling along with the nurturing of home life. A significant part of this work in creating a 'proper' home involves the continual ordering of time, spaces, and resources into configurations to solve ongoing problems of living. To facilitate the orderings and routines of homemaking, a plethora of technologies are used. Indeed, Western homes function through the use of products, tools, machines, gadgets, and equipment-from toothbrushes to door locks. Homes are metamachines of literally thousands of different technological components.

Our contention is, as we have detailed above, that the nature of some of these material technologies in terms of everyday objects is changing as they increasingly become infused with software. Domestic objects are gaining capacities that extend their technicity and enable them to do additional work in the world. Indeed, it seems likely that the majority of objects that currently use electrical power will become colonised by computer code in the (near) future, just as a wide range of manual and mechanical household tools became newly animated by the development and integration of electrical control and motors in the first half of the 20th century (Cowan, 1983). These capacities, we argue, are helping to reshape domestic living and its spatialities by, on the one hand, augmenting and supplementing domestic tasks and, on the other, plugging the home into new, extended, distributed networks. In other words, coded objects are reconfiguring the social and material relations of home, often in banal and subtle ways. They do so, we argue, because they transduce space: that is, they beckon new spaces and spatialities into being through their actions. Coded objects make anew a domain, such as a home, in reiterative and transformative practices through the work that they perform (Mackenzie, 2003). Significantly, they can do this without human oversight by processing information they have generated or received and determining courses of action.

(8) Although not for all, as illustrated by the extent of domestic violence (cf Warrington, 2001).

(9) According to the UK 2005 Time Use Survey, on average people in Britain spend $70 \%$ of their time at home (ONS, 2006). Around one third of this time is spent sleeping. 
As we have detailed previously, coded objects beckon two particular forms of space into being - code/space and coded space (Dodge and Kitchin, 2004). Code/spaces are spaces dependent on software to function. That is, the relationship is dyadic. Without software-enabled technologies the space would not be produced as intended. For example, a home office that requires an Internet connection to enable a person to check e-mail and work on files remotely is dependent on code to produce the intended spatiality. If the computer or the Internet connection 'fails' then the space fails to be a remotely extended home office. Coded space, on the other hand, is a spatial transduction that is mediated by coded processes, but whose relationship is not dyadic. In other words, software-enabled technologies produce particular spatialities, but if they are not present or operative a space is still produced as intended but less efficiently or cost-effectively. For example, if a software-controlled burglar-alarm system fails, the house is still a secure home, albeit a less safe one than the householder planned. Most digital objects in a household transduce a home as coded space-that is, they make a difference to how the space is transduced, but they are not essential to the majority of domestic tasks and the function of most rooms (there are alternative means of solving domestic problems by configuring other resources and home spaces in necessary ways, such as for cooking and cleaning, personal care, and providing entertainment).

Here, we are interested in coded objects in the home, but it should also be noted that home is represented by and worked upon by a wide range of coded processes external to material dwelling spaces (see also Dodge and Kitchin, 2005a). Consumer and governmental instruments of measurement, surveillance, and classification concerning, for example, personal finances, insurance, taxation, utilities, and welfare benefits, envelope households in multiple, overlapping grids of calculation; nearly all of this is now undertaken using databases and processed automatically by software algorithms (cf Graham, 2005; Lyon, 2002). In the case of geodemographic representations homes are 'sorted' according to perceived value or risk and the results of this sorting determine which services are offered to them (cf Parker et al, 2007).

\section{Home-coding vignettes}

To illustrate our taxonomy and argument so far, in this section we document coded objects from three 'typical' British homes. These vignettes are based on broad observation of different homes, but should be acknowledged as being fictional. Their aim is purely illustrative and, despite the anecdotal empiricism employed, we believe they highlight how a range of kinds of coded objects are now commonly embedded in millions of homes and are widely used to solve a host of different domestic tasks. Of course, the vast majority of homes still possess many objects working in analogue form, demonstrating that the transition to something approaching 'pervasive computing' occurs faster and slower depending on the person, place, and circumstance (cf Rode, 2006). Furthermore, we acknowledge the somewhat gendered nature of technology use in the vignettes, which clearly does not reflect the multiplicity of situations in many homes, but is still common within the manufacturing and marketing of domestic technologies (Whitehead, 2008; also evident in figure 2). In the vignettes, PCO refers to a peripherally coded object; HC to a hard codeject; $\mathrm{CC}$ to a closed codeject; SC to a sensory codeject; PL to a permeable logject; and NL to a networked logject; see also figure 1).

\section{Vignette 1}

Peter (aged 43 years), Wendy (aged 40 years) and their three children (Joshua, 10; Toby, 4; Milly, 1) constitute a typical 'hard-working family'. They live in a mortgaged, three-bedroom, semidetached house in a mixed private-public housing estate. 
Both parents work full-time (in manual and lower managerial occupations) and they have a hectic home life raising the children. A range of technologies and an increasing number of coded objects are used in the daily production of their home, creating a series of overlapping coded space and code/space, as a basic audit of areas of their house reveals.

\section{Living room}

The main family room is the focus of entertainment and information gathering, and an intense point of digital media use at different times of the day. Pride of place in the room is given over to a large flat-screen digital television (CC) partnered with a coterie of coded objects to provide it with apposite media sources. The family have recently upgraded from a separate analogue video recorder and DVD player to an integrated digital video recorder (PL). A Sky+ satellite decoder box (NL) is connected to the telephone line: this is necessary to access some of the more sophisticated interactive services, particularly pay-per-view sports enjoyed by the family. The move to a digital television system has increased the range of viewing options and enhanced flexibility of schedules. Together the televisual assemblage works as a powerful networked logject which 'watches' the family and communicates the log to their service provider-which can then build up a profile of their viewing habits and preferences. Code renders the television a two-way mirror which watches the viewers as the viewers watch it.

One corner of the living room has been semipermanently reconfigured to serve as a computing 'zone' for the main family desktop PC (along with printer, scanner, and various 'connectors' for cameras, PDA, and the like). It is permanently connected to the Internet via broadband, and is the most obvious networked logject in the home, revealing much of their domestic online activities to their ISP (Internet service provider) (cf Bennett, 2001). At the moment, the digital camcorder, an expensive and relatively little used permeable logject, is on the table next to the PC. The living room also plays host to an Xbox360 video-game console (PL), which can be connected to the Internet and contains a multifunction cordless phone and answer machine which serves as main contact 'number' for the home, but is being usurped increasingly by the flexibility offered by individual family members' own mobile phones (NLs).

\section{Kitchen - diner}

Along with the living room, the kitchen is typically the busiest room in the house, serving various home functions such as preparing food, cooking, washing, ironing, and socialising. These functions are often aided by domestic appliances, many new models of which are now augmented by code. In Peter and Wendy's case, most of their appliances are more than five years old and are analogue or peripherally coded in nature: cooker/hob (PCO), dishwasher, fridge - freezer, and tumble dryer. As such, they are largely uncoded objects, with electromechanical or electronic controls, but are likely to become coded objects when next replaced. This has already occurred with respect to the washing machine (SC), which is a new model that offers a raft of software-driven programs and options from its LED control panel. While the software potentially makes a difference to the wash, in this case the family typically only uses a couple of preset programs so that the code makes little, if any, difference to the household's laundry practices. In one corner of the kitchen is the control panel for the central heating (SC), which was recently updated when the boiler was replaced. The control is code driven, notionally providing much greater control over heating (timings and temperature level), with settings held in a rudimentary database. 


\section{Bedrooms}

These rooms are typically more private spaces and are less coded than the living room and kitchen. The master bedroom contains several pieces of home gym equipment which include digital performance monitors (PL), used on a semiregular basis by Peter. Joshua's attic bedroom contains a growing number of coded objects, including a new laptop computer, a DVD player, an MP3 player and speakers, a digital camera, and what he perceives as an aging video-games console that cannot play the latest games (all PL unless consciously networked). The room also has other media technologies, including an analogue television and radio. Toby's bedroom has a large range of toys, some of which use electronics and software to provide interactive features (HCs and $\mathrm{CCs}$ ). The fourth bedroom is serving as a nursery for baby Milly, and contains a baby sleep monitor and electronic learning toys which often are activated by software (HCs and SCs).

\section{Bathroom}

The family bathroom is the most private space in the home and also the least mediated by electrical and electronic-powered technologies. Yet it is a highly technological space, one that is dominated by machinery to channel water safely and remove waste efficiently. As a pivotal space for personal care of the body, it contains several portable coded objects, including Peter's digital 'body monitoring' weighing scales (PL) and Wendy's pedometer (PL).

\section{Vignette 2}

Simon (aged 43 years) and Iris (aged 37 years) are both full-time, professional workers with no children or pets. They live in a two-bedroom apartment in a new city-centre complex, with attendant security gates, key pads, and CCTV cameras. They have a high disposable income, are technologically savvy, time pressured, and security conscious. They regularly work at home as part of their lifestyle. Their apartment is actively monitored by a building-wide fire-and-smoke alarm system, and monitored externally by coded services provision in terms of metered gas, electricity, water, cable broadband Internet, and cable television.

\section{Living room}

The living room contains a variety of very expensive, branded home-entertainment technologies (all are coded objects). A large high-definition plasma screen television (HC) is mounted on one wall, linked to a home-theatre amplifier and surround-sound speakers. It is also connected to a cable television set-top box (NL), a DVR (PL), and to a Slingbox (PL), a wireless networking device that distributes the digital television signal into other rooms of the apartment. Simon and Iris are music aficionados and the room has a state-of-the-art hi-fi system which is heavily coded but does not log usage and is not networked. On the table is a one-year-old Apple MacBook laptop (NL) which Iris and Simon use as a home PC for surfing the web, including online grocery shopping; it is always networked via Wifi to the household cable broadband. On the mantelpiece are two Kodak Easyshare digital picture frames (CC), showing a sequence of photographs from their summer holiday in New Zealand. They have no fixed line telephone: they use the cable broadband for Wifi and VoIP (voice over Internet protocol) calls (using Skype software), and have multiple mobile phones (for work and personal use). 


\section{Kitchen}

The space is purposefully designed as a 'luxury' fitted kitchen with top of the range appliances and a built-in media centre (LCD-liquid crystal display-television and DVD player), all of which are unitary coded objects and are programmable in some way. The kitchen also has a DAB (digital audio broadcasting) radio (CC), although the

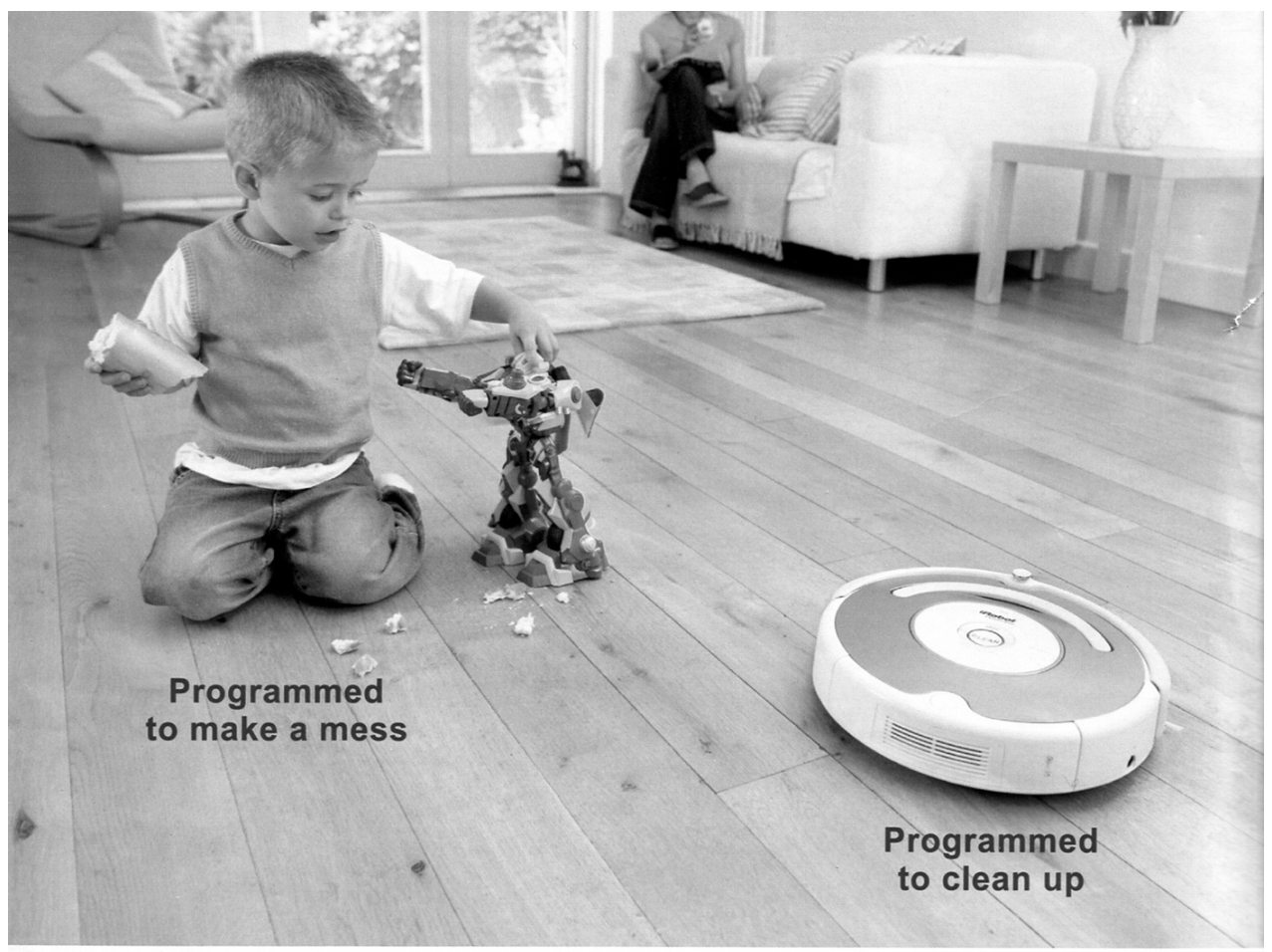

\section{iRobot Roomba: cleans routinely...so you don't have to}

The new floor vacuuming robot that really works

Keeping on top of the cleaning and floor cleaning in particular is a constant battle in any home. Thankfully, the new iRobot Roomba is designed to relieve you of this tedious chore and help you on a daily basis. It cleans floors superbly at the press of a button, using less energy than a standard vacuum.

How does iRobot Roomba work? Advanced sensors and AWARE ${ }^{\circledR}$ robot technology ensure this intelligent and energy efficient home robot covers your whole floor area. Whether it's carpets, rugs or hard floor surfaces, its highly effective brush system and smart vacuum picks up large debris as well as fine dust and dirt. It even gets right under most furniture to clean those difficult to reach areas.

Just turn it on, walk away and come back to clean, mess free floors.

For more information on iRobot Roomba call 0800132509 Ormotec
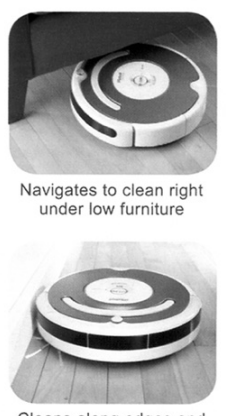

Cleans along edges and in tight corners

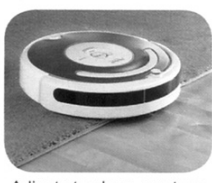

Adjusts to clean any type of floor surface

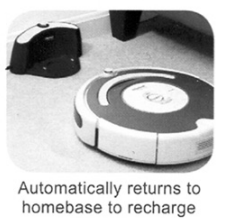

www.irobot.com

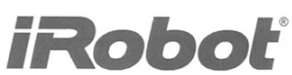

Figure 2. Magazine advertisement for domestic coded object that promises greater convenience and leisure through automation. It is also an overtly gendered representation of technology and domestic practice with the messy boy playing while a passive mother figure in the background undertakes the childcare (source: Guardian weekend magazine 20 October 2007, page 34. Reproduced by kind permission of iRobot). 
addition of software makes little difference to Simon's radio listening whilst cooking. On one wall is an LCD panel which provides the software interface to control the environmental system (NL) for the apartment, which offers individual room heating and air-conditioning profiles, along with wireless connection and remote online access. In a cupboard, next to the vacuum cleaner, is a redundant coded gadget that Simon bought for Iris as a humorous present (figure 2). This robotic convenience (SC) has only been a used a couple of times as they pay a cleaner to come in and clean the apartment two mornings a week.

\section{Bedrooms}

Their main bedroom has little coded technology except for an alarm clock - radio (CC) and an LCD digital television and Slingbox receiver for cable television signal (PL). The room is often a transitory site for various mobile codejects (such as phones, PDAs, laptops, MP3 players) at different times. The second bedroom of the apartment is permanently configured as a home office and contains a range of computers and associated paraphernalia to support Simon and Iris when they want to work from home. There is a new desktop PC and an aging iMac (both NL) along with a laptopdocking station and monitor (HC), plus cradles for a PDA and an iPod (HC). On top of one of the monitors is a webcam (NL) that Simon uses occasionally. Under the desk is a wireless router (NL) for the cable broadband that provides secure Wifi networking throughout the apartment; also hidden away there is a redundant fax machine (HC) fully replaced by the PC and now inoperable as the apartment does not have a conventional landline phone connection. The bottom of a filing cabinet also contains several generations of digital cameras (SC), a camcorder (PL), a couple of redundant mobile phones (NL) and media players (PL), and an external hard disk (PL) for infrequently made backups of their growing range of personal data. On top of the filing cabinet is a wireless colour laser printer (NL). Lastly, the office is the storing place for Simon's large digital keyboard synthesiser (PL) and the GPS (PL) that Iris uses for geocaching at weekends.

\section{Entrance hallway}

Next to the front door of the apartment is a video entry phone (CC) which allows them to control visitor access into the building. Nearby is the control panel for the apartment's burglar alarm (NL) that is connected to a central monitoring station. (There are four movement sensors at different points in the apartment, along with sensors on several windows that are potentially vulnerable). The storage cupboard in the hallway also contains two 'smart meters' (NL), recording the household's utility usage.

\section{Vignette 3}

Dorothy (aged 85 years) has lived in the same two-bedroom terraced house for over forty years and has been a widow for eight years. She has a limited fixed-pension income. She is still relatively active and enjoys visits from her grandchildren, but she has a growing number of medical problems which have reduced her mobility. She has come to rely on homecare and assistive, 'quality of life' technologies and telemonitoring to enable her to keep living independently, but much of her house is uncoded.

\section{Living room}

In the living room there is a limited set of home-entertainment technologies including a television (analogue), VCR (CC), and a radio-CD player (HC). From television adverts Dorothy is aware of a 'digital switch over' (the phased termination of analogue television broadcasts across the UK) which means that she will need to update her 
television set, but is worried about costs and uncertain over the details of what she will need to do. There is a cordless digital telephone base station (HC) on the coffee table, and Dorothy usually takes the handset with her as she moves about the house during the day as otherwise she risks missing phone calls. She has no mobile phone. The living room also contains the Lifeline control box for the telecare home-monitoring system (NL) that unobtrusively 'watches' Dorothy's daily activities and provides a safety net for summoning help if she has an incident in the house. This box is permanently networked via a landline phone connection to a remote control centre. It is wired to passive infrared (PIR) movement sensors in all rooms and to several fixed panic alarms (including a pull cord in the bathroom) as well as the pendant alarm (HC) that Dorothy wears.

\section{Kitchen}

The kitchen contains a range of older electrical appliances, none of which are coded but which remain perfectly functional. The central heating control is an old 'clockwork' timer with a manual thermostat. The exception is a new DAB radio $(\mathrm{CC})$ on the kitchen counter, received as a Christmas present; it is permanently tuned into Dorothy's favourite local station and its additional functionality ignored. A basic calculator (CC) is on the table on top of a couple of utility bills, and the day's post lies on a counter, including a bulky padded envelope containing her repeat prescription of tablets ordered automatically for her by a health-management database at the local pharmacy. The post also contains a couple of pieces of junk mail calculated by the geodemographic profile for this postcode to appeal to typical householder, but quite inappropriate for Dorothy's lifestyle. Dorothy's home and domestic activities, relatively uncoded at the immediate scale of the dwelling, are nonetheless still represented and automatically worked upon by software at various distant sites.

\section{Bedroom and bathroom}

These rooms have no coded objects beyond the PIR movement sensors on the wall, a panic alarm button/cord and a bed-occupancy sensor-a pressure pad under the mattress (HC).

\section{Software practices and the spatialities of home}

These vignettes highlight that whilst the type and number of coded objects vary, software is already prevalent in Western homes. Furthermore, homes are being networked into a range of coded processes that distanciate domestic practices and open them up to routinised monitoring and profiling. Coded objects alter the material, social, and spatial relations of the home in new ways: they offer members of households new affordances to undertake domestic living differently: to record television programmes when they are away from the home, to have more choice of programme, and to watch them at different locations about the house; to source information or purchase goods without leaving the home; to cook food for a set time without being present in the kitchen; to play new kinds of games and with people located at some distance; to enjoy photographs and music in new ways; to be monitored for health from a distance; to work at home whilst being in constant contact with the office and to move from room to room whilst doing so; and so on. Digital technologies are different from their analogue equivalents, which might have performed similar roles, in several important ways: they offer more functionality; they are more interactive; they are often programmable; they work independently of human oversight; many can record their use; some can communicate with other devices and with information systems across networks. 
Code thus makes a difference to the nature of domestic living by enabling a variety of digital technologies to augment, supplement, and replace analogue technologies, as well as providing new kinds of technology that undertake novel tasks. In so doing, coded objects make a difference to the transduction of home space: how the spatiality of the home is beckoned into being as coded space or code/space. Their supplementary capacities provide additional, partial solutions to the relational problems of domestic living (for example, cleaning, cooking, entertaining, personal care) and enable other problems to be addressed from the home (such as undertaking the management of household finances, work-related tasks, health monitoring).

For example, the computers and broadband connection in Simon and Iris's apartment transduces the space into a site of work: both are able to undertake work-related tasks at a distance whilst being connected in real time to their workplaces' servers. The apartment is spatially re-configured to facilitate such a transduction, with a bedroom converted into an office. The PC reduces the time required to undertake tasks such as editing, redrafting, and sending a document, and transforms where these tasks can be undertaken. Similarly, the games computer in Peter and Wendy's home transduces the space of the living room into a node on a global network across which people can play games in real time with opponents distributed around the world. The digital television, set-top box, and DVR alter how the living room is transduced into a space of entertainment by enabling a flexibility of choice of television content that can be watched at their leisure, as opposed to the schedule of the broadcasters. Simon's digital keyboard transduces the space of his office into a music studio where he is able to compose, edit, record, and play back multilayered and instrumented songs. Dorothy's Lifeline control box transduces her home into a site of continuous yet unobtrusive healthcare monitoring that enables her to live at home rather than having to move to sheltered housing. The environmental system in Simon and Iris's apartment transduces the space into one with a comfortable climate which responds immediately to changes in temperature and humidity. These spatial transductions are only possible through the local application of software which ties the home into wider networks and myriad layers of coded processes.

The home is also spatially reconfigured by coded objects by the deepening and widening of dwellings as nodes in a variety of networks - utilities, entertainment, health, communications. Homes have long been ensnared in such networks, but information flow was usually unidirectional and what was done with the information was confined to the home (for example, television programmes were beamed into the home but what was watched was unknown other than viewing figures extrapolated from small-panel audience sampling). Through coded objects, homes are being embedded in real-time, two-way networks so that the everyday nature of domestic practices (which programmes one watches and records, who one talks to on a phone and for how long, what one looks at or purchases on the Internet, how one uses electricity and water) can be monitored by service providers with the attendant data being used to profile and social sort customers. The consequence of networked logjects is that homes are increasingly being stretched out across space in networks of greater and shorter length - and, as such, scaled in new ways - and they are subject to increasing levels of (corporate) surveillance.

\section{Pervasive computing and the promise of the 'home of the future'}

For many technologists, the embedding of coded objects into homes is evidence that we are moving to the era of the 'smart home' and widespread pervasive computing. Pervasive computing, as defined by Galloway (2004, pages $384-385$ ), "seeks to embed computers into our everyday lives in such ways as to render them invisible and allow them to be taken for granted." The aim of its advocates is to augment all aspects of 
everyday life and activities through the addition of computing power to objects and spaces, rendering them smart to some degree, and yet also mundane and routine (Dodge and Kitchin, 2007b).

Given the growing range of digital technologies and software in the home, it is not surprising that within pervasive-computing research the domestic sphere is a target of much investigation and speculation (cf Bell and Dourish, 2007; Crabtree et al, 2003; Edwards and Grinter, 2001; Taylor and Swan, 2005). There is a belief that, as Galloway (2004) argues, if computers can be brought wholesale into our home worlds then domestic practices can be radically altered. A central trope in such research is the notion of a home that "anticipates and responds to the needs of the occupants, working to promote their comfort, convenience, security and entertainment through the management of technology within the home and connections to the world beyond" (Aldrich, 2003, page 17). Such anticipation and response will be fully automated, automatic, autonomous, decided upon by sophisticated software algorithms designed to be reflexive to home users' desires and wishes. This is the vision of the 'smart home' - a home with computing power built into all the objects contained within; a home that is aware of itself and of its past activity, its surroundings, its inhabitants, its contents, and its external service providers, and knows how to react appropriately to different scenarios. This vision anticipates computing power being built not just into objects within the home, but also into the fabric of the dwelling itself.

With respect to coded objects in particular, some in the pervasive computing community presently envisage their logical end point as spimes (Sterling, 2005). A 'spime' is a wholly new kind of object, for which there is an entire recorded history stretching from manufacture to disposal. Such histories will include 'deep' details on: (1) everything used to make, process, and distribute that object, plus protocols for safe and sustainable disposal; (2) everyone/everything that has come into contact with that thing during its lifetime; and (3) the context of making and use-labour relations, cost and profit margins, carbon tax, patents. In other words, a spime is an object that has a full genealogy wherein the entire actor network of a thing is knowable and indexical, which, Sterling (2005, page 11) asserts, means that they are "material instantiations of an immaterial system ... [they] begin and end as data."

Although no spimes exist at present, there are projects and programmes which are developing what might be might be termed 'protospimes': that is, they invest objects with spime-like capacity, although their capacities exist external to the thing being recorded. For example, there have been a number of projects to make transparent the full extent of food production (cf Popper, 2007). With respect to agriculture, these are moving beyond existing 'farm-to-fork' tracking systems to much more granular tracing that aims to follow livestock from conception (that is, recording both parents and, over time, the lineage of all animals and how they were reared) to the consumer's home (through farms, abattoirs, processor and manufacturers, logistics chains, and supermarkets). A smart home would consist of an assemblage of computationally rich building fabric and spimes.

While it is possible to argue that we are on the path to such an assemblage, it must also be recognised that smart homes are a particular sociotechnical vision developed by technologists: the latest reincarnation of a long-running modernist fantasy of technology capable of producing orderly domestic spaces and maximising leisure time, often based on gendered stereotypes of domestic practice (figure 2; cf Corn and Horrigan, 1984; Whitehead, 2008). Indeed, the premise of a smart home has been common across several generations of home design (Spigel, 2005), promulgated by a nexus of product designers, housebuilders, and appliance manufacturers, and, increasingly, the software industry, focused on driving new rounds of consumer fashions and home 'upgrades'. 
The domestication of software also clearly has a variety of potential social implications. Perhaps the most obvious is the scope for greater control of mundane and personal activities occurring in the home and a concomitant impact on freedom and privacy. Networked logjects, in particular, open up the home through continuous flows of data, potentially rendering unseen domestic activities and previously personal behaviours visible to corporations. This control by code will vary from subtle, almost voluntarist, conditioning that is little noticed, such as the preselection of potentially interesting programmes to watch by a television, or 'body monitoring' bathroom scales chiding the user for missing their target weight and urging greater efforts of performance (cf Schuurman, 2004), to a more potent form where coded objects refuse to perform because they determine that an action is 'illegal' (for example, copyright enforcement through digital-rights management stopping the computer playing movies not legally owned [see Dodge and Kitchin (2005b), Graham (2005), and Lyon (2002) for a fuller discussion on the enrolment of software in the surveillance, classification, and control of consumptive practices].

Yet, control is not the whole story. At the same time, code opens up genuinely novel avenues for creative solutions to domestic tasks - particularly in terms of pleasure and play. How software can make things differently is well illustrated by new children's toys and games, typically HCs and CCs but increasingly also SCs. Indeed, creating fun is an important conduit through which software is seeping deeper into the sinews of home life. As Thrift (2003, page 400) notes, toys are "rapidly becoming something else: something between a lumpen object onto which all manner of fantasies and all kinds of play could be projected and a kind of alternative life form, participating in the world on at least some terms of its own choosing."

In addition to the spectre of control and empowerment of creativity, the enrolment of code on a wide scale into the home brings with it a whole new layer of complexity and risks to daily living, despite the rhetoric of software making life easier. A foretaste of this complexity is the real cognitive work required in maintaining home PCs and mobile devices in proper order. It is estimated that several million compromised home PCs are presently connected to the Internet (Leyden, 2005), in large part because their owners are technically unable or unwilling to invest time to keep them patched and protected with updated software and to keep passwords secure. As more and more everyday domestic tasks are undertaken with network logjects, it will become increasingly important to maintain them. The result will be the development of a whole new domain of 'digital housekeeping' (Crabtree et al, 2007) to keep software-driven appliances stable and relatively secure.

The complexity of code will also be felt in the form of excessive functionality, where a previously simple task achieved with a straightforward dial or a couple of selection switches becomes overwhelmed by menus, options, and check boxes on-screen. It is likely that many people will simply fall back on default settings that 'seem to work', a point echoed with earlier rounds of 'complex' electronic home technology like the VCR, where large numbers of people failed to be able to program them successfully and simply used them as basic playback devices (cf Rode et al, 2004). Greater complexity also entails risks, particularly where coded objects become the primary (and perhaps only) store of household information (such as significant financial records or sentimentally valuable photographs). The risks of relying on software to keep these digital media safe is compounded because people are often poor at maintaining systematic backups or any backup at all (a very tedious piece of digital housekeeping), and where the coded object becomes permeable these media become potentially vulnerable to unauthorised remote access and theft. 
Given these various issues, there will, almost certainly, be some populations who actively resist the growing encroachment of software into the home, concerned by the potential for surveillance by outside parties, along with those who will 'hack' the code to subvert the deadweight of 'technological paternalism' (Spiekermann and Pallas, 2006), and those who will voice objections because of the frustration caused by function overload and excessive, software-induced, complexity.

The extent to which 'smart homes' as envisaged by technologists will come to fruition is, therefore, doubtful. While it is evident that code is increasingly becoming part of everyday homes and does make a difference to how domestic practices unfold, as illustrated by our vignettes, any transition to the era of the 'smart home' will take place over a long period of time. With respect to the adoption of coded objects, many homes continue to contain analogue objects that will in many cases be used until they need to be replaced. In other cases, coded objects are expensive luxury items that require a certain income, lifestyle, and technical literacy to purchase and operate. With respect to the development of computationally rich building fabric this will require extensive and expensive retrofitting of existing buildings that is unlikely to be undertaken without significant benefits to the home dweller or external regulatory pressure (such as requirements of mortgage lenders/insurers, or waste/energy reduction in the name of more sustainable living). At present, it is unlikely that such adaptations will offer such tempting benefits, especially with the rapid redundancy that currently accompanies technological change. As with the take-up of any set of technological innovations, the adoption of coded objects into the home will be uneven and unequal, both socially and geographically - dependent on person, place, and circumstance.

\section{Conclusion}

In this paper we have argued that everyday objects, in use in a range of domestic settings, are increasingly becoming coded and folded into the 'Internet of things'. To start to make sense of this diverse range of coded objects we first provided a taxonomy based on their relational capacities. Peripherally coded objects are objects in which software has been embedded, but such code is not essential to their use. Codejects are dependent upon code to function. Hard codejects rely on code to function, but are not programmable. Unitary codejects rely on software to function, but do not keep a record of their work in the world for future reuse. We identified two classes of unitary codejects, closed codejects, which act independently of the world around them; and sensory objects that sense and react to environments. Logjects have an 'awareness' of themselves and their relations with the world and automatically record aspects of those relations in ways that can inform future activity. We identified two classes of logjects: permeable logjects are functionally self-contained units which can work fully and log their use independently of any wider network; and networked logjects that do not function without continuous access to transmission networks.

We then argued that these types of coded objects make homes differently. Often working in autonomous ways, coded objects transduce into being different spatial formations - coded space and code/space. While the transduction of code/space is relatively rare in domestic settings, code is certainly central to how many domestic tasks are now performed, with the transduction of coded spaces common. In other words, the everyday use of coded objects reshapes the spatiality of the home by altering how domestic tasks are undertaken (not always more conveniently for all), introducing new tasks and sometimes greater complexity, and embedding the home in diverse, extended systems of consumption and governmentality. How coded objects beckon space into being is not, however, deterministic: rather, it is contingent and relational; it varies across place, time, and context (see Dodge and Kitchin, 2005a). The spatiality 
of different homes, even if they were identical in every way, would vary substantially because the technologies would be used in different ways within varying contexts. These contexts are social and familial, but are also structured within the wider political economy (for example, market-led pricing, fragmentation of consumer-service contracts), legal arrangements and standards (for example, health and safety), evolving cultural practices (for example, when and where it is acceptable to use certain coded objects), and differential access to certain services based on social sorting (cf Lyon, 2002).

To illustrate our arguments we examined three 'typical' British homes. These homes revealed that a diverse range of coded objects are already present within all the spaces of the home. In some cases these coded objects have already become mundane and slipped into the background 'technological unconscious' (Thrift, 2004), and yet they perform vital roles in holding together household routines. Arguably, in Dorothy's case the monitoring of her by coded objects and software algorithms ensures a greater degree of safer living which in combination with other sociotechnical supports means that she can remain living in her own home. In other cases the coded objects are seen as novel and are feted as technological breakthroughs which provide new ways of being and acting. Indeed, many homes now contain multiple iterations of the same appliances (particularly those for entertainment), along with older and superseded versions (rendered obsolete by new functionality or mere changes in consumer fashion). Some others are perhaps little more than gimmicks, such as continued attempts to provide robotic solutions to domestic drudgery (figure 2).

As we have documented, the transition into the fully software-enabled home is a slow, incremental process. As such, homes presently contain a mixture of electrical, electronic, and coded technologies which are enrolled together daily to solve tasks of living and beckon home spaces in being. Homes, then, are made through an imperfect but normally functional bricolage of coded components. Rather than making the domestic realm more orderly, the infusion of software into homes is leading to a new 'overcoding' of routines and activities which often makes home life more complex and prone to unexpected and inexplicable failure and disruptions.

We are confident, however, that we are at a juncture in the production of home space, as domestic objects become more and more coded-either through software being embedded into their makeup, or as machine-readable objects embedded in the 'Internet of things'. While this is an incremental not epochal change, we would also argue that a useful parallel can be drawn between the contemporary coding of homes and the initial domestication of electricity at the end of the 19th century. At first, electrical power was a merely an expensive novelty (for example, electric light versus gas lighting) and initially there were few electrical appliances. Over an extended period of time, existing technologies were converted to use electricity (open hearth to electric heater, range to electric cooker, washtub to washing machine, etc), with small electrical motors being used to replace manual labour, and a raft of new domestic tools produced [some of the most potent but now mundane being the refrigerator and freezer, which have significantly changed domestic practices relating to food consumption (cf Hand and Shove, 2007; Watkins, 2006)]. The extent to which electricity powers almost everything of significance in our homes today is largely unremarked in a Western context (except in a power cut), and homes are necessarily built so that electricity supply is available in every space.

Our belief is that software has comparable social-technical agency to that of electricity, driving technologies that work both in the foreground and in the background to shape domestic living and spatiality in all kinds of unconscious ways. Whereas electrical motors replaced physical labour, software algorithms will supplement and 
augment human decision making. As such, we feel that coded objects demand further attention as key future domestic actants. While we have made a start in this paper to sketch out their emerging forms and the work that they perform in the world, over time there will be a need to examine more fully their nature from an ontological and epistemological point of view and to tease out the difference they make across a number of domains such as home, workplaces, and public spaces, and to fundamental spatial processes such as communication and mobility. For us this will need to entail the construction of detailed ethnographies of the development, use, and networking of different kinds of coded objects; how they are placed into and become key actants in complex actor-networks; and how they work in diverse conjunctions with people to realise a multiplicity of spaces and spatialities.

\section{References}

Albrecht K, McIntyre L, 2005 Spychips: How Major Corporations and Government Plan to Track Your Every Move with RFID (Nelson Current Publishers, Nashville, TN)

Aldrich F K, 2003, "Smart homes: past, present and future", in Inside the Smart Home Ed. R Harper (Springer, London) pp $17-39$

Beer, D, 2007, "Thoughtful territories: imagining the thinking power of things and spaces" City $11229-238$

Bell G, Dourish P, 2007, "Back to the shed: gendered visions of technology and domesticity" Personal and Ubiquitous Computing 11373 - 381

Bennett C J, 2001, "Cookies, web bugs, webcams and cue cats: patterns of surveillance on the world wide web" Ethics and Information Technology 3(3) 195-208

Bleecker J, 2006, "Why things matter: a manifesto for networked objects-cohabiting with pigeons, arphids and aibos in the Internet of things", http://www.nearfuturelaboratory.com/ files/WhyThingsMatter.pdf

Blunt A, Dowling R, 2006 Home (Routledge, London)

Blunt A, Varley A, 2004, "Introduction: Geographies of home" Cultural Geographies 11 3-6

Corn J J, Horrigan B, 1984 Yesterday's Tomorrows: Past Visions of the American Future (Johns Hopkins University Press, Baltimore, MD)

Cowan R S, 1983 More Work for Mother: The Ironies of Household Technology from the Open Hearth to the Microwave (Basic Books, New York)

Crabtree A, Rodden T, Hemmings T, Benford S, 2003, "Finding a place for ubicomp in the home" Lecture Notes in Computer Science $2864208-226$

Crabtree A, Tolmie P, Rodden T, Greenhalgh C, Benford S, 2007, "Making the home network at home: digital housekeeping" Proceedings of the 10th European Conference on ComputerSupported Cooperative Work Limerick, http://www.mrl.nott.ac.uk/ axc/documents/papers/ ECSCW_2007.pdf

Crang M, Graham S, 2007, "Sentient cities: ambient intelligence and the politics of urban space" Information Communication and Society $10789-817$

Dodge M, Kitchin R, 2004, "Flying through code/space: the real virtuality of air travel" Environment and Planning A 36 195-211

Dodge M, Kitchin R, 2005a, "Code and the transduction of space" Annals of the Association of American Geographers 95162 - 180

Dodge M, Kitchin R, 2005b, "Codes of life: identification codes and the machine-readable world" Environment and Planning D: Society and Space 23851 - 881

Dodge M, Kitchin, R, 2007a, "The automatic management of drivers and driving spaces" Geoforum $38264-275$

Dodge M, Kitchin R, 2007b, “'Outlines of a world coming in existence': pervasive computing and the ethics of forgetting" Environment and Planning B: Planning and Design 34431 - 445

Edwards W K, Grinter R E, 2001, "At home with ubiquitous computing: seven challenges" Proceedings of the Conference on Ubiquitous Computing (Ubicomp 2001) Atlanta, GA, http://www.cc.gatech.edu/ keith/pubs/ubicomp2001-challenges.pdf

Galloway A, 2004, "Intimations of everyday life: ubiquitous computing and the city" Cultural Studies $18384-408$

Graham S D N, 2005, "Software-sorted geographies" Progress in Human Geography 29562 - 580

Hand M, Shove E, 2007, "Condensing practices: ways of living with a freezer" Journal of Consumer Culture $779-104$ 
Hitchings R, 2004, "At home with someone nonhuman" Home Cultures 1169 - 186

Leyden J, 2005, "Rise of the botnets" The Register 15 March, http://www.theregister.co.uk/2005/03/ 15/honeypotbotnetstudy/

Lyon D, 2002, "Everyday surveillance: personal data and social classifications" Information, Communication and Society $5242-257$

Mackenzie A, 2003, “Transduction: invention, innovation and collective life", http://www.lancs.ac.uk/ staff/mackenza/papers/transduction.pdf

Mallett S, 2004, "Understanding home: a critical review of the literature" The Sociological Review $52156-179$

Miller D, 2001 Home Possessions: Material Culture Behind Closed Doors (Berg, Oxford)

Mitchell W J, 2005 Placing Words: Symbol, Space and the City (MIT Press, Cambridge, MA)

Murtaugh M, 2008, "Interactivity”, in Software Studies: A Lexicon Ed. M Fuller (MIT Press, Cambridge MA) pp $143-149$

Nova N, Bleecker J, 2006, "Blogjects and the new ecology of things" Lift06 Workshop http://tecfa.unige.ch/-nova/blogject-lift06.pdf

ONS, 2006 The Time Use Survey, 2005 Office for National Statistics, London, http://www.statistics.gov.uk/articles/nojournal/time_use_2005.pdf

Parker S, Uprichard E, Burrow R, 2007, "Class places and place classes: geodemographics and the spatialization of class" Information, Communication and Society $10902-921$

Pollock N, Williams R, 2008 Software and Organisations: The Biography of the Enterprise-wide System or How SAP Conquered the World (Routledge, London)

Popper D E, 2007, "Traceability: tracking and privacy in the food system" Geographical Review $97365-388$

Rode J A, 2006, "Appliances for whom? Considering place" Personal and Ubiquitous Computing 10(2/3) $90-94$

Rode J A, Toye E F, Blackwell A F, 2004, "The fuzzy felt ethnography-understanding the programming patterns of domestic appliances" Personal and Ubiquitous Computing 8161 - 176

Schoenberger C R, 2002, "The internet of things" Forbes Magazine 18 March, http://www.forbes.com/technology/forbes/2002/0318/155.html

Schuurman N, 2004, "Databases and bodies: a cyborg update" Environment and Planning A 36 $1337-1340$

Spiekermann S, Pallas F, 2006, “Technology paternalism - wider implications of ubiquitous computing" Poiesis and Praxis: International Journal of Ethics of Science and Technology Assessment 4 6-18

Spigel L, 2005, "Designing the smart house: posthuman domesticity and conspicuous production" European Journal of Cultural Studies 8405 - 426

Sterling B, 2005 Shaping Things (MIT Press, Cambridge, MA)

Taylor A, Swan L, 2005, "Artful systems in the home", in Proceedings of the Conference on Human Factors and Computing Systems (CHI '05) (ACM Press, New York) pp $641-650$

Thrift N, 2003, "Closer to the machine? Intelligent environments, new forms of possession and the rise of the supertoy" Cultural Geographies $10389-407$

Thrift N, 2004, "Movement - space: the changing domain of thinking resulting from the development of new kinds of spatial awareness" Economy and Society $33582-604$

Thrift N, French S, 2002, "The automatic production of space" Transactions of the Institute of British Geographers, New Series 27 309-335

Warrington M, 2001, “'I must get out': the geographies of domestic violence" Transactions of the Institute of British Geographers, New Series $26365-383$

Watkins H, 2006, "Beauty queen, bulletin board and browser: rescripting the refrigerator" Gender, Place and Culture $13143-152$

Whitehead M, 2008, "Domesticating technological myth: gender, exhibition spaces and the clean air movement in the UK" Social and Cultural Geography $9635-651$ 
Conditions of use. This article may be downloaded from the E\&P website for personal research by members of subscribing organisations. This PDF may not be placed on any website (or other online distribution system) without permission of the publisher. 